\title{
SIMULATION OF HELICOPTER ROTORS ON UNSTRUCTURED MIXED MESHES USING EDGE-BASED RECONSTRUCTION SCHEMES
}

\author{
Vladimir Bobkov, Ilya Abalakin, Tatiana Kozubskaya \\ Keldysh Insitute of Applied Mathematics \\ 4A, Miusskaya Sq., Moscow, 125047, Russia \\ vbobkov@keldysh.ru, http://caa.imamod.ru
}

Key words: Helicopter Rotor, Unstructured Mixed Mesh, Edge-Based Reconstruction Scheme, EBR

\begin{abstract}
The paper gives a detailed description of the developed numerical method for simulating the turbulent flow around a helicopter rotor and calculating its aerodynamic characteristics. The method is based on the original vertex-centered finite-volume EBR schemes. The features of these schemes are their higher accuracy that is achieved through the use of edge-based reconstruction of variables on extended quasi-one-dimensional stencils, and a moderate computational cost that allows for serial computations. When dealing with discontinuities or solutions with large gradients, a quasi-one-dimensional EBR-WENO scheme is used. The methods are implemented in the in-house code NOISEtte within the hybrid MPI-OpenMP parallel model. Validation of the developed techniques is carried out by simulating several cases for hovering and forward-flight regimes of helicopter rotors.
\end{abstract}

\section{INTRODUCTION}

The methods of mathematical modelling and numerical simulation are being actively introduced into industrial applications in the aviation industry. Numerical prediction is becoming a powerful tool for developing the optimal configurations of aircraft and determining the optimal operating modes. To make a computational experiment practically useful in the design of a helicopter rotor, it is necessary to build a correct mathematical description of all essential gas-dynamic processes and to develop accurate and not too expensive numerical methods, which makes it possible to carry out fast predictions on available supercomputers.

Almost all existing and implemented in commercial software packages methods for calculating the aerodynamic and acoustic characteristics of helicopter rotors use multi-block structured meshes and ensure the accuracy not higher than of the second order. The method described in this work and implemented in the in-house code NOISEtte [1] implies a complete gas-dynamic description of the medium in a noninertial rotating coordinate framework, by which we mean the systems of differential equations built based on the Navier-Stockes equations for a viscous compressible gas for describing turbulent flow. In this paper, we consider mostly RANS methods, however hybrid RANS-LES approaches are also implemented in NOISEtte.

There are set of studies devoted to rotor aerodynamics numerical simulation. In papers $[2,3]$ the results of solving similar problems using numerical modeling, where a system of Reynolds-averaged equations 
Navier-Stockes, closed by various turbulence models, is chosen as a mathematical model to describe the motion of a viscous turbulent flow of a compressible gas. Currently for the rotors aerodynamics modeling the high order schemes on the mixed-element unstructured grids are used with compressible RANS equations [4].

In the paper we are concentrated mostly at the numerical method we developed earlier for unstructured hybrid meshes and now apply for simulating turbulent flow over a helicopter rotor. The method is based on the original vertex-centered finite-volume EBR (Edge-Based Reconstruction) schemes which provide higher-accuracy of the numerical result thanks to the underlying quasi-one-dimensional edge-oriented reconstructions of flux variables [5, 6].

To validate the developed method, we represent the results of simulating several cases considering a helicopter rotor both in hovering mode and forward flight.

\section{MATHEMATICAL MODEL}

\subsection{Navier-Stockes equations in non-inertial frame of reference}

The gas flow around a rotor rotating at an angular velocity $\omega$ is calculated using the system of Reynoldsaveraged Navier-Stokes equations closed by the Spalart-Allmaras turbulence model. The system of equations is considered in a noninertial rotating reference frame. The coordinate axes rotate around the fixed rotor axis at a constant angular velocity equal to that of blade rotation. This means that the rotor blades in a flow are fixed, while the upstream flow direction changes depending on the azimuthal angle
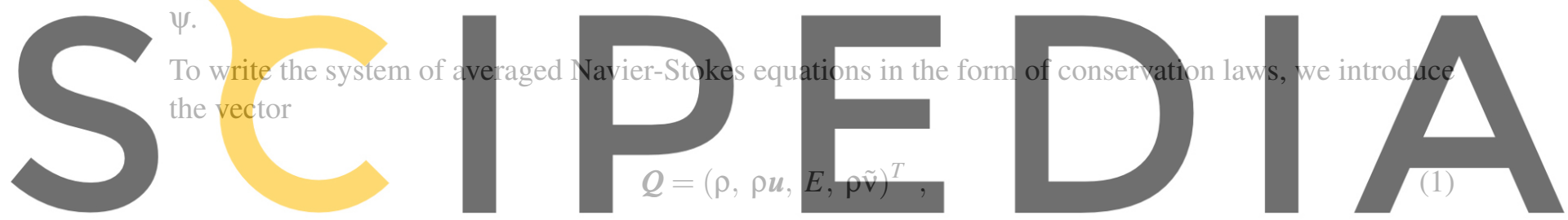

where $u=\left(u_{1}, u_{2}, u_{3}\right)$ - is the velocity vector in the fixed reference frame, $\rho$ is the density, $E=\rho u^{2} / 2+$

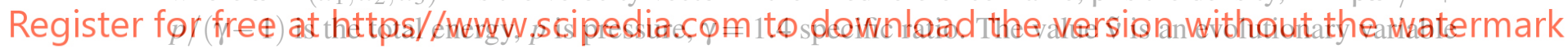

necessary to determine the turbulent viscosity coefficient $\mu_{T}$ in the Spalart-Allmaras model:

$$
\mu_{T}=\rho \tilde{v} \frac{\chi^{3}}{\chi^{3}+357.911}, \chi=\frac{\rho \tilde{v}}{\mu}
$$

where $\mu$ is molecular dynamic viscosity coefficient.

We also introduce the vector of the linear velocity of rotation $\boldsymbol{V}=\left(V_{1}, V_{2}, V_{3}\right)^{T}=\boldsymbol{\omega} \times \boldsymbol{r}$ determined by the angular velocity vector $\boldsymbol{\omega}$ and the position vector $\boldsymbol{r}$.

Then the system of averaged equations Navier-Stockes in a noninertial rotating reference frame can be written in the following pseudovector form ${ }^{1}$

$$
\frac{\partial \boldsymbol{Q}}{\partial t}+\nabla \cdot\left(\mathcal{F}^{C}(\boldsymbol{Q})-\mathcal{F}^{R}(\boldsymbol{Q})-\mathcal{F}^{D}(\boldsymbol{Q}, \nabla \boldsymbol{Q})\right)=\boldsymbol{S}(\boldsymbol{Q}, \nabla \boldsymbol{Q})
$$

\footnotetext{
${ }^{1}$ A brief derivation of the system of equations Navier-Stockes for a compressible gas in a non-inertial rotating reference frame and its component-wise form is given in the work [7].
} 
Composite vectors $\mathcal{F}^{C}, \mathcal{F}^{R}$ and $\mathcal{F}^{D}$ are introduced in the system (2). The components of this vector $\boldsymbol{F}_{i}^{C}$, $\boldsymbol{F}_{i}^{R}$ and $\boldsymbol{F}_{i}^{D}$ are the convective transport flux vector, the rotational transport vector and the diffusion flux vector in coordinate direction $x_{i}(i=1,2,3)$. The operator $(\nabla \cdot)$ is the divergence operator applied to each component of the composite vector.

The fluxes vectors of convective transport and rotational transport fluxes are set as functions of the physical variables $\rho, \boldsymbol{u}, p$ as follows

$$
\begin{aligned}
& \boldsymbol{F}_{i}^{C}(\boldsymbol{Q})=\left(\rho u_{i}, \rho u_{i} \boldsymbol{u}+p \boldsymbol{I},(E+p) u_{i}, \rho \tilde{\mathbf{v}} u_{i}\right)^{T}, \\
& \boldsymbol{F}_{i}^{R}(\boldsymbol{Q})=\left(\rho V_{i}, \rho u_{i} \boldsymbol{V}, E V_{i}, \rho \tilde{\mathbf{v}} V_{i}\right)^{T},
\end{aligned}
$$

where $\boldsymbol{I}$ is the identity matrix. The diffusion flux vectors are determined as functions of physical variables and their gradients by the formula

$$
F_{i}^{v}(\mathbb{Q}, \nabla \mathbf{Q})=\left(0, \tau_{i 1}, \tau_{i 2}, \tau_{i 3}, \tau_{i j} u_{j}+q_{i}, \frac{3}{2}(\mu+\rho \tilde{v}) \frac{\partial \tilde{\mathbf{v}}}{\partial x_{i}}\right)^{T}
$$

where the components of the viscous stress tensor $\tau_{i j}$ and the heat flux vector $q_{i}$ have the form

$$
\tau_{i j}=\left(\mu+\mu_{T}\right)\left(\frac{\partial u_{i}}{\partial x_{j}}+\frac{\partial u_{j}}{\partial x_{i}}-\frac{2}{3} \delta_{i j} \frac{\partial u_{i}}{\partial x_{i}}\right), q_{i}=\left(\frac{\mu}{\operatorname{Pr}}+\frac{\mu_{T}}{\operatorname{Pr}_{T}}\right) \frac{\partial \varepsilon}{\partial x},
$$

Here $\delta_{i j}$ is Kronecker symbol, $\mu$ is the molecular viscosity coefficient, $\operatorname{Pr}=0.72, \operatorname{Pr}_{T}=1$ are molecular and turbulent Prandtl numbers.

The vector $S(Q, \nabla Q)$ is a source term describing transport of the main variables $Q$
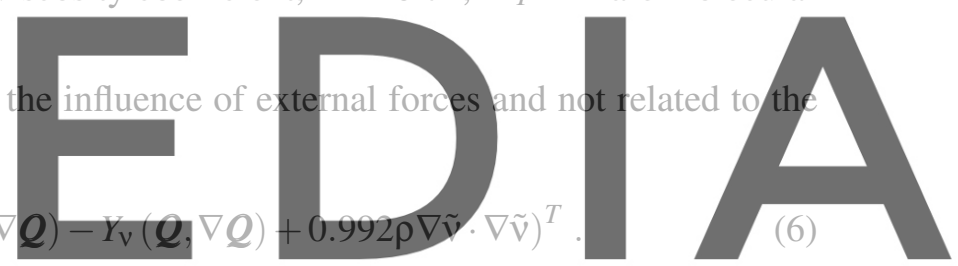

Register for free at hthe gradient of the variable v is a part of the definition of the diffusion term in the last equation of the system(3), which has a nondivergence form, and therefore is transferred to the source term.

From the standpoint of an observer in the fixed reference frame the system of equations (3)-(6) describes the variation in the conservative variables at the expense of their convective and diffusion transport in the medium rotating at the velocity $\boldsymbol{V}$, the pressure gradient, and the vector velocity rotation through the azimuthal angle $\psi(t)=-|\boldsymbol{\omega}| t$.

\subsection{Aerodynamic characteristics}

In the problems of numerical modelling of a helicopter rotor some of the main parameters characterizing the properties of a configuration are the aerodynamic coefficients, namely, the thrust and torque coefficients.

We will define the aerodynamic forces of a blade as follows. We will consider two reference frames, one of which, $(x, y, z)$ is fixed and the other, $\left(x^{\prime}, y^{\prime}, z^{\prime}\right)$, is fitted to the rotating rotor. Since the calculation is performed in the reference frame rotating about the $O z$ axis (Fig. 1), the axes of the fixed and rotating coordinate systems can be identified. Thus, the noninertial rotating reference frame is characterized by a 

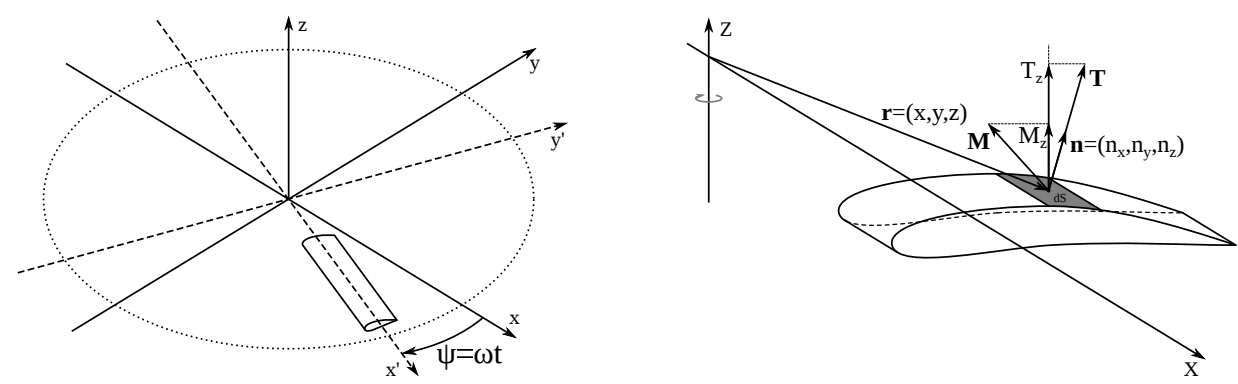

Figure 1: Rotating frame of reference (left) and blade aerodynamic forces scheme (right)

rotation of the fixed reference frame through an angle $\psi(t)+\pi / 2$ around the $O z$ axis, where the azimuthal angle $\psi(t)$ is defined as $\psi(t)=-|\boldsymbol{\omega}| t=-\boldsymbol{\omega} t$.

The resultant force vector $\boldsymbol{R}$, acting on the blade, and the resultant moment $\boldsymbol{M}$ are determined by the surface integrals

$$
\boldsymbol{R}=\oint(p \boldsymbol{n}-\tau \cdot \boldsymbol{n}) d s, \boldsymbol{M}=\oint \boldsymbol{r} \times(p \boldsymbol{n}-\tau \cdot \boldsymbol{n}) d s,
$$

where $p$ and $\tau$ are the pressure and viscous stress tensor distributions over the rotor surface $S$, vector $n$ is the external unit normal to the surface $S$ with components $n_{x}, n_{y}, n_{z}$ (Fig. 1), $r$ is the position vector(torque vector).

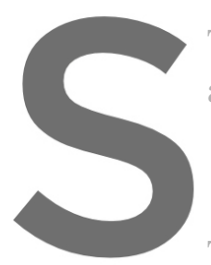

The rotor thrust $T$ is defined as t as follows

The rotor foree moments are determined as the proj
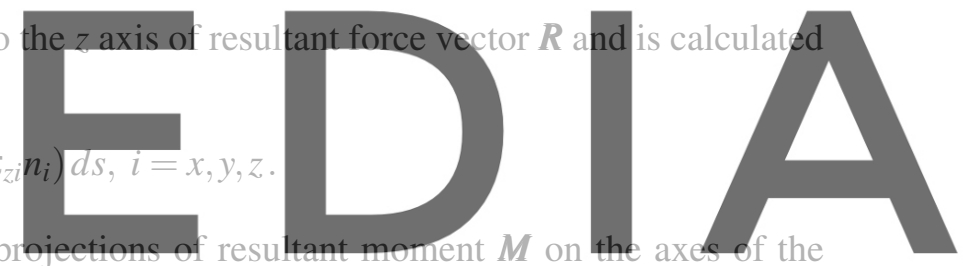

fixed reference frame $(x, y, z)$. Thence follows the expression for the aerodynamic torque moment of the

Register fordiree at https//www.scipedia.com to download the version without the watermark

$$
M_{k}(t)=\oint\left[x\left(p n_{y}-\tau_{y i} n_{i}\right)-y\left(p n_{x}-\tau_{x i} n_{i}\right)\right] d s, i=x, y, z .
$$

The thrust coefficient $c_{T}$ and the aerodynamic torque coefficient $m_{k}$ are calculated by nondimensionalizing the thrust and torque values

$$
c_{T}(t)=\frac{2 T(t)}{\rho_{0} A(\omega R)^{2}}, \quad m_{k}(t)=\frac{2 M_{k}(t)}{\rho_{0} A(\omega R)^{2} R},
$$

where $\rho_{0}$ is the undisturbed air density, $A=\pi R^{2}$ is the rotor disk area, $R$ is the rotor radius, $\omega$ is the absolute value of the angular velocity of a blade.

We also introduce the normal force coefficient $c_{n}$ determined in the normal section of the blade $L$

$$
c_{n}=\frac{1}{\rho_{0} A(\omega R)^{2}} \int_{L} p n_{z}^{\prime} d l,
$$

Here $n_{z}^{\prime}=n_{x}^{\prime} \cos \varphi-n_{z}^{\prime} \sin \varphi$ is the component of the normal vector in the blade fixed reference frame. 


\section{NUMERICAL METHOD}

\subsection{Edge-Based Reconstruction Schemes}

Spatial discretization in the software package NOISEtte is based on a vertex-centered formulation with the setting of variables at the nodes of the hybrid grid $^{2}$ and the determination of numerical fluxes on the faces of the computational cells surrounding the nodes. The finite volume method is used to approximate convective fluxes of the Navier-Stokes equations. The finite volume is computational cell.

The approximation of the system of equations Navier-Stockes (2) is based on the integral form of this system. Let us write the system (2) in discrete form for the computational cell $K_{i}$ (Fig. 2) surrounding the mesh node $i$

$$
\int_{K_{i}} \frac{d \boldsymbol{Q}}{d t} d V+\int_{\partial K_{i}}\left(\mathcal{F}^{C}(\boldsymbol{Q}) \cdot \boldsymbol{n}-(\boldsymbol{V} \cdot \boldsymbol{n}) \boldsymbol{Q}\right) d S=\int_{K_{i}} \nabla \cdot \mathcal{F}^{D}(\boldsymbol{Q}, \nabla \boldsymbol{Q}) d V+\int_{K_{i}} S(\boldsymbol{Q}, \nabla \boldsymbol{Q}) d V
$$

where $\partial K_{i}$ is the boundary of the computational cell $K_{i}, n$ is unit outward normal to the boundary $\partial K_{i}$. Then the finite volume method defines the following spatially discrete equation

$$
\frac{d \boldsymbol{Q}_{i}}{d t}=-\frac{1}{\left|K_{i}\right|} \sum_{j \in N_{1}(i)} \boldsymbol{h}_{i j}+\boldsymbol{S}\left(\boldsymbol{Q}_{i},(\nabla \boldsymbol{Q})_{i}\right), \quad \boldsymbol{h}_{i j}=\boldsymbol{h}_{i j}^{C, R}+\boldsymbol{h}_{i j}^{D}
$$

where $\left|K_{i}\right|$ is the volume of the computational cell $K_{i}, N_{1}(i)$ is the set of first-level neighbours of node

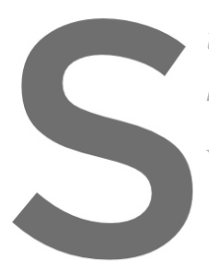

$i, h_{i j}$ is numerical flux

$h_{i j}^{C, R}$ and $h_{i j}^{D}$ of convective

We calculate $h_{i j}^{C, R}$ by Rde solver
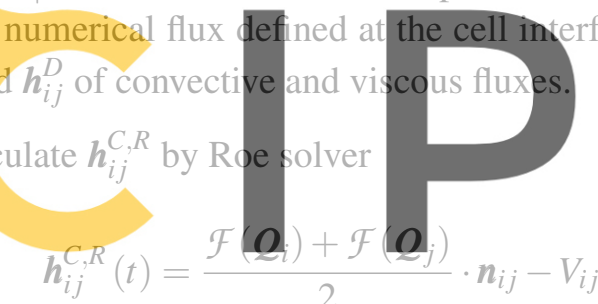

$\frac{\left.Q_{i}+Q_{j}\right)}{2}-\frac{\delta}{2} S^{-1}$

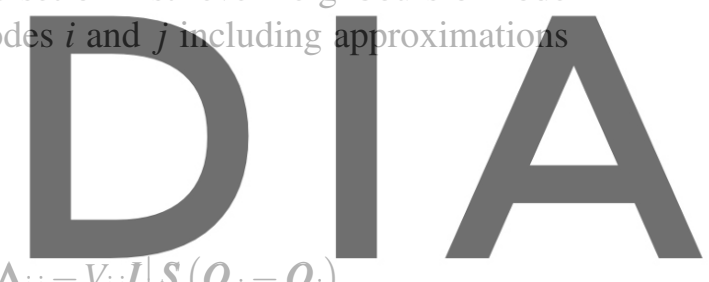

Register for free at https//www.scipediancom to download the dersion without the watermark

$$
n_{i j}=\int_{\partial K_{i}(t) \cap K_{j}(t)} n d S, \quad V_{i j}=\int_{\partial K_{i} \cap K_{j}} V \cdot n d S, \quad A_{i j}^{C}=\frac{d \mathcal{F}}{d \underline{Q}}\left(Q_{i j}\right)=S_{i j} \Lambda_{i j} S_{i j}^{-1}
$$

where $\boldsymbol{A}_{i j}$ is the Jacobian depending on the vector of conservative variables $\boldsymbol{Q}_{i j}$ defined on the interface (or the middle of the edge) $i j, \boldsymbol{\Lambda}_{i j}$ is the diagonal matrix of the eigenvalues of the Jacobian $\boldsymbol{A}_{i j}, \boldsymbol{n}_{i j}$ is the vector area of the surface determined by the common boundary of control volumes $K_{i}$ and $K_{j}$, $\delta$ is the numerical dissipation parameter.

To increase the order of approximation of the numerical convective flux (7), a quasi-1D edge-based reconstruction of variables on an extended stencil is used [8]. This approach involves replacing the variables $\boldsymbol{Q}_{i}$ and $\boldsymbol{Q}_{j}$ in (7) with the values $\boldsymbol{Q}_{i j}^{L}$ and $\boldsymbol{Q}_{i j}^{R}$ reconstructed to the left and right of the cell edge. Matrices $\boldsymbol{S}$ and $\boldsymbol{\Lambda}$ do not change. The method of calculating the values of $\boldsymbol{Q}_{i j}^{L}$ and $\boldsymbol{Q}_{i j}^{R}$ on the stencil

\footnotetext{
${ }^{2}$ The hybrid mesh consists of the following elements - hexahedra, triangular prisms (in the boundary layer region), tetrahedrons, and quadrangular pyramids (outside the boundary layer).
} 

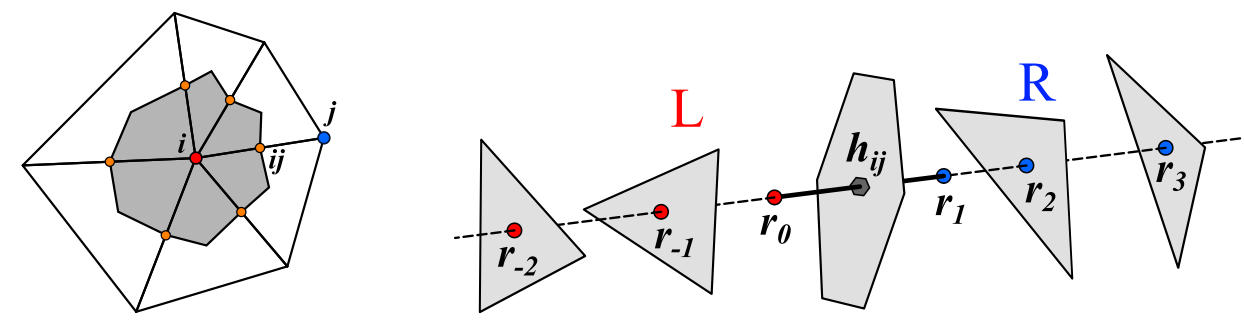

Figure 2: Example of median control volume for triangular mesh(left) and 3D space stencil for EBR5 scheme(right)

$\left\{S_{i j}^{(n, 0)}\right\}$ defined at $2 n+2$ points $\left\{\boldsymbol{r}_{k}\right\}(k=-n, \ldots, n+1)^{3}$ determines a specific edge-based scheme $\operatorname{EBR}(2 \mathrm{n}+1)$ [5]. The values at points $\left\{\boldsymbol{r}_{k}\right\}$ that do not coincide with the mesh nodes are determined by linear interpolation on the corresponding faces of the mesh elements that intersect with the straight line containing the edge $i j$ (Fig. 2).

The EBR5 scheme was used to perform the calculations presented in this article.

\subsection{Time advance}

The time integration is performed using an implicit second-order scheme followed by the Newtonian linearization of the space-discretized system of equations. At each Newtonian iteration stage the corresponding system of linear equations is solved by BiCGSTAB method.
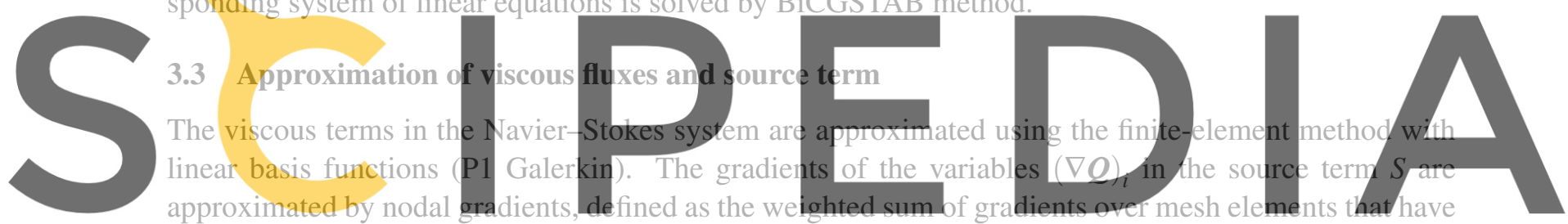
a common vertex at node $i$.

Register for free at https//www.scipedia.com to download the version without the watermark 4 NUMERICAL RESULTS

\subsection{Caradoma-Tung rotor}

The first validation case was modeling the aerodynamic characteristics of a two-bladed Caradonna-Tung rotor in hovering mode [11]. Where is a lot of experimental data for the case including per-section pressure coefficient distribution and tip vortex evolution.

The model rotor is two-bladed rigid rotor with rectangular planform blades with no twist or taper. The blades are based on the NACA0012 airfoil with fixed collective pitch $8^{\circ}$, rotor radius is $1.143 \mathrm{~m}$, blade chord $-b=0.1905 \mathrm{~m}$. The rotational speed in hovering mode was 650,1750 and 2300 RPM with corresponding tip Mach number $\mathrm{M}=0.23,0.61$ and 0.8 . The corresponding Reynolds number evaluated by blade chord and tip velocity was $\operatorname{Re}=0.98,2.65,3.48 \times 10^{6}$.

Due to the hovering mode and rotor symmetry it was possible to model only the single blade with periodic

\footnotetext{
${ }^{3}$ Here the local indexing of reconstruction nodes is introduced: node $i$ corresponds to index 0 , node $j$ corresponds to index 1. This indexing defines $n$ interpolation points of the reconstruction located to the left of the node $i$ and $n$ points located to the right of the node $j$.
} 

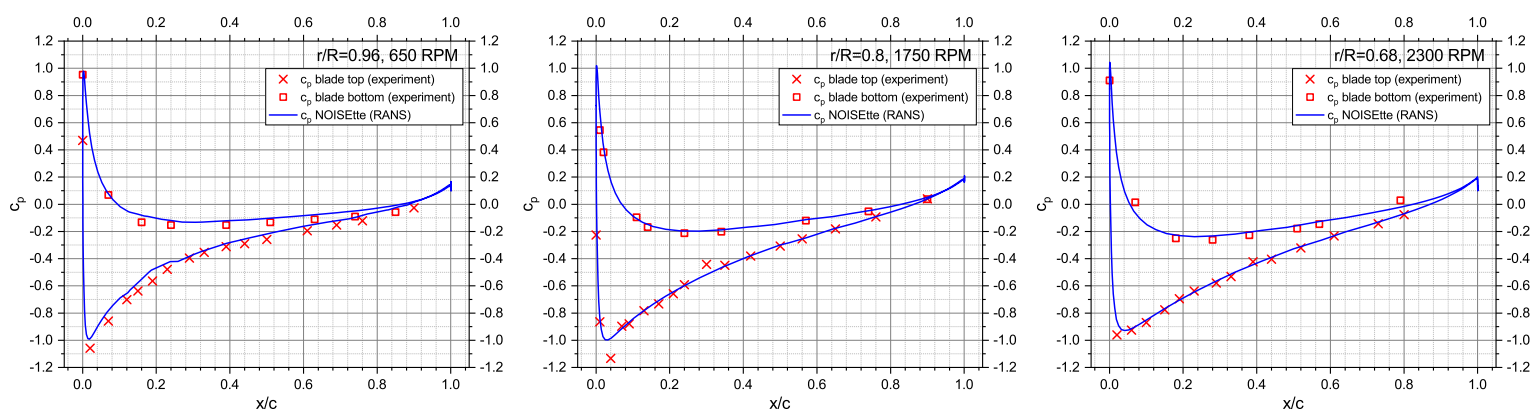

Figure 3: Caradonna-Tung pressure coefficient distribution at the blade sections $r / R=0.96,0.8$ and 0.68

boundary conditions on the azimuthal planes. A RANS approach with the Spalart-Allmaras model has been used in the simulation.

Here and in the following cases the mixed mesh generated in the same way: firstly the unstructured surface mesh refined at the blade leading and trailing edges and at the tip is build, than the prismatic boundary layer with constantly increasing prism height is build. When the prism sizes turns to isotropic the rest computational domain filled with tetrahedrons.

For the Caradonna-Tung rotor simulation the mixed unstructured mesh with $1.37 \mathrm{M}$ nodes and $5.6 \mathrm{M}$ cells was used.

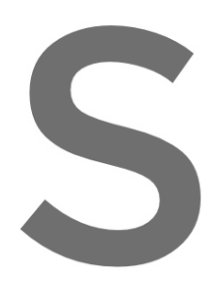

Comparison of numericat

span-wise locations def

agreement with the exp
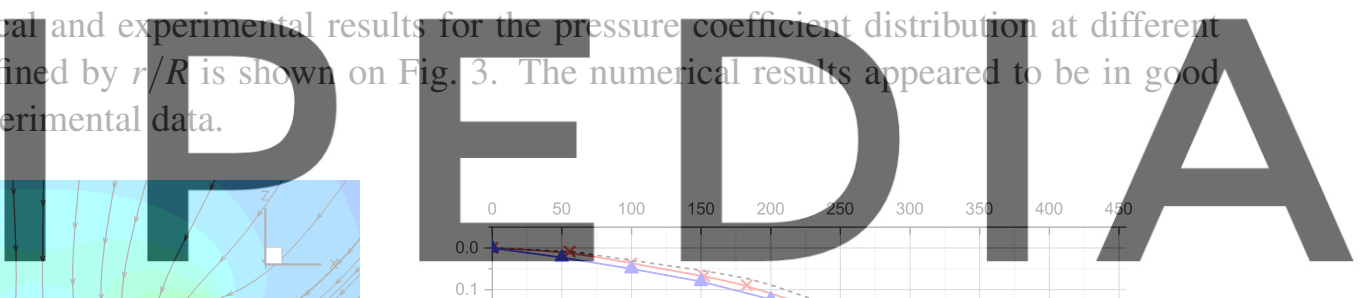

Register for free at https//www.scipedia.com
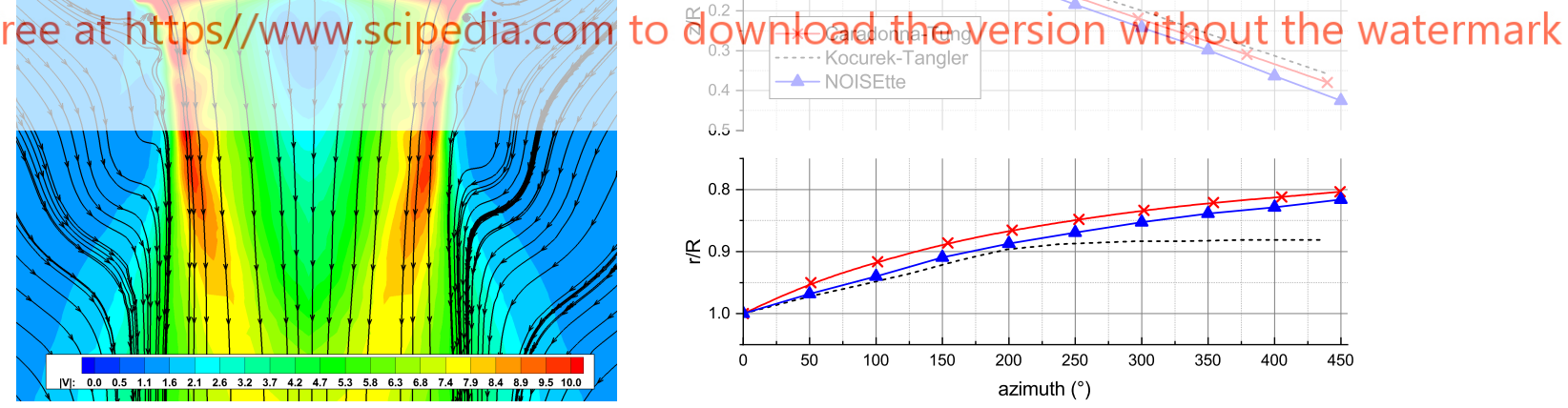

Figure 4: Velocity field (left) and trajectory of tip vortex core (right) for rotation velocity 650 RPM

Fig. 4(left) represents the typical velocity field and streamlines in $x O z$ section. As expected the flow velocity maximum reached near the blade tip and flow velocity decreased downstream to the rotor disk. The position of the tip vortex core (see Fig. 4(right)) is in good agreement with both the experimental data and the Kocurek-Tangler analytical prediction [12]. 


\subsection{Rigid four-bladed rotor in hover}

As the second validation problem, the problem of axial flow around the model helicopter rotor was chosen in accordance with a physical experiment conducted at the Department of Aerohydrodynamics Kazan National Research Technical University named after A. N. Tupolev (DA KNRTU-KAI).

The goal of the numerical experiment was to estimate pressure fluctuations near the model four-bladed rotor (hereinafter referred to as the "model rotor"), tested in a wind tunnel T-1K with an acoustic noiseabsorbing chamber in DA KNRTU-KAI.

In the experiment, pressure pulsations in the near field were measured using an array of microphones (see Fig. 5). An array grid with a 150mm step, consisting of three lines of 13 microphones each, was located at a distance of $208 \mathrm{~mm}$ from the edge of the rotor disk in such a way that the top line of the microphones lay in the rotor plane, and the central microphone ("mic06" on Fig. 5) was closest to the rotor axis of rotation.
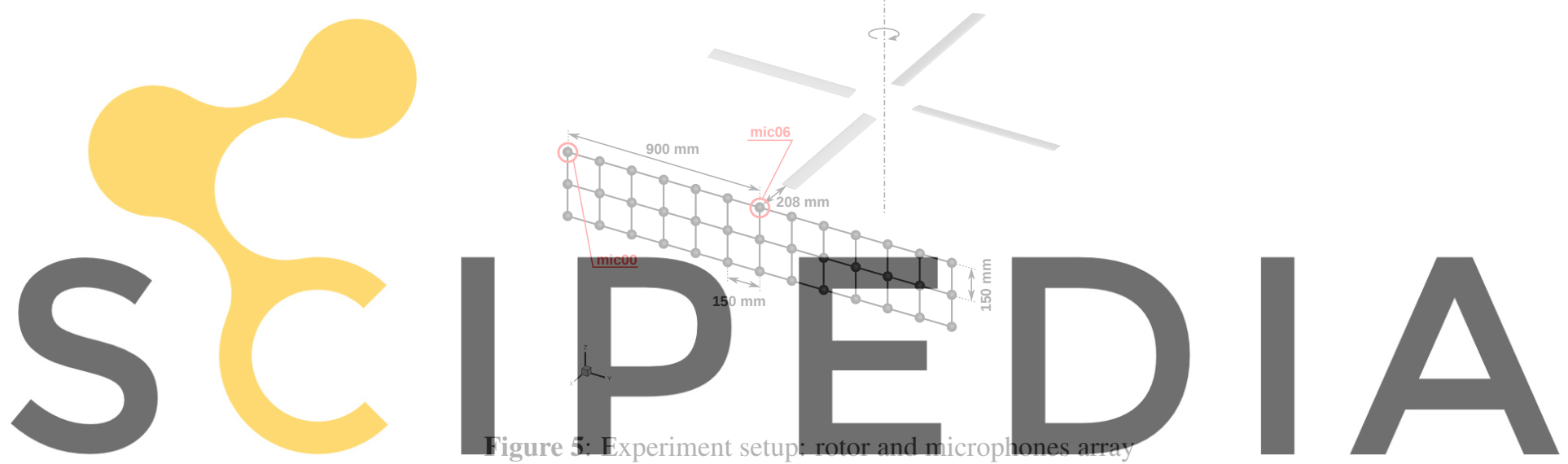

 \\ setup. The rigid untwisted four-bladed rotor with radius $0.8 \mathrm{~m}$ and blade chord $0.065 \mathrm{~m}$ in hover with}

911RPII speed was modeled. Blades shape based on the NACA0012 airfoil with fixed collective pitch $8^{\circ}$. The value of the Reynolds number evaluated by the chord of the blade and its tip velocity $V_{\text {tip }}=76.34 \mathrm{~m} / \mathrm{s}$ was $\operatorname{Re}=0.322 \times 10^{6}$.

Due to the hovering mode and rotor symmetry it was possible to model only the single blade with periodic boundary conditions on the azimuthal planes. A RANS approach with the Spalart-Allmaras model has been used in the simulation. For the rotor simulation the mixed unstructured mesh with 3.7M nodes and $17 \mathrm{M}$ cells was generated and used with EBR5 numerical scheme.

The calculated pressure pulsations measured at the minimum (probe "mic06") and maximum (probe "mic00") distance from the rotor plane (see Fig. 6) shows good agreement with signals measured within the experiment. 

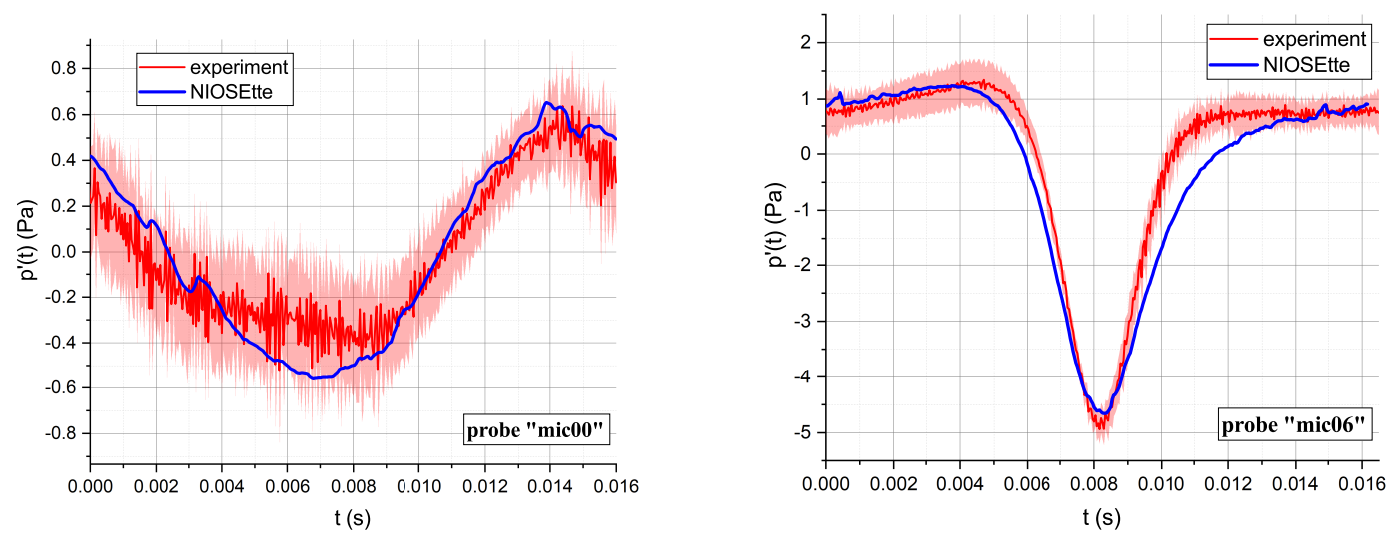

Figure 6: Pressure pulsations in the probes "mic00"(left) and "mic06"(right)

\subsection{Shrouded rotor}

The next challenging problem was to model the axial flow and determine the aerodynamic characteristics of a model rotor in a ring - it represents the Fenestron-type tail rotor scheme. The problem setup is notable due to presence of both moving aerodynamic surfaces (rotor blades) and the fixed one (ring surfaces).

The problem setup reproduces the experiment setup performed in Kamov construction bureau: a small scaled rigid four-bladed rotor rotates inside a toroidal ring. The speed of rotation is 1166.4 RPM with

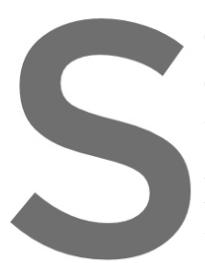
corresponding tip velocity chord was $0.13 \mathrm{~m}$. The vath velocity was $\operatorname{Re}=0.628$

Due to the axial flow regirne and shrouded rotor symmetry it was $p$ with single blade with peri
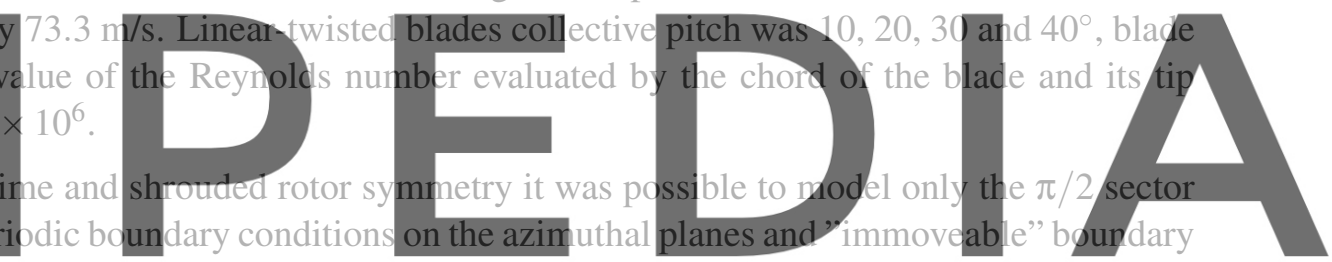

conditions on the ring. For the shrouded rotor simulation the set of mixed unstructured meshes was

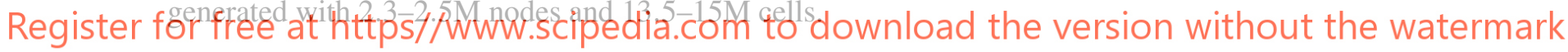

The rotor aerodynamic characteristics evaluated within computational experiments perfectly aligned with the experimental results (see Fig. 7). It confirms the correctness of modeling a complex problem that includes both rotating and stationary elements.

The full description of the problem setup and results represented in the [13, 14].

\subsection{Rigid rotor in forward flight mode}

In this study, the validity of the numerical simulation of the flow around rotating helicopter rotor is confirmed by means of comparing the results of the calculations of a model rigid main rotor in forward flight.

The modeled configuration corresponds that tested in a series of wind tunnel tests performed in the Central Aerohydrodynamic Institute (TsAGI) and represents the main rigid four-blade helicopter main rotor with a set of plane untwisted blades of rectangular planform, rigid in bend and torsion. The blades are based on the NACA-0012 airfoil with the blade chord $0.15 \mathrm{~m}$ with $8^{\circ}$ collective pitch. The rotor radius is $1.2 \mathrm{~m}$ while the rotor hub is an ellipsoid of revolution with the horizontal and vertical radii $0.04 \mathrm{~m}$ and 

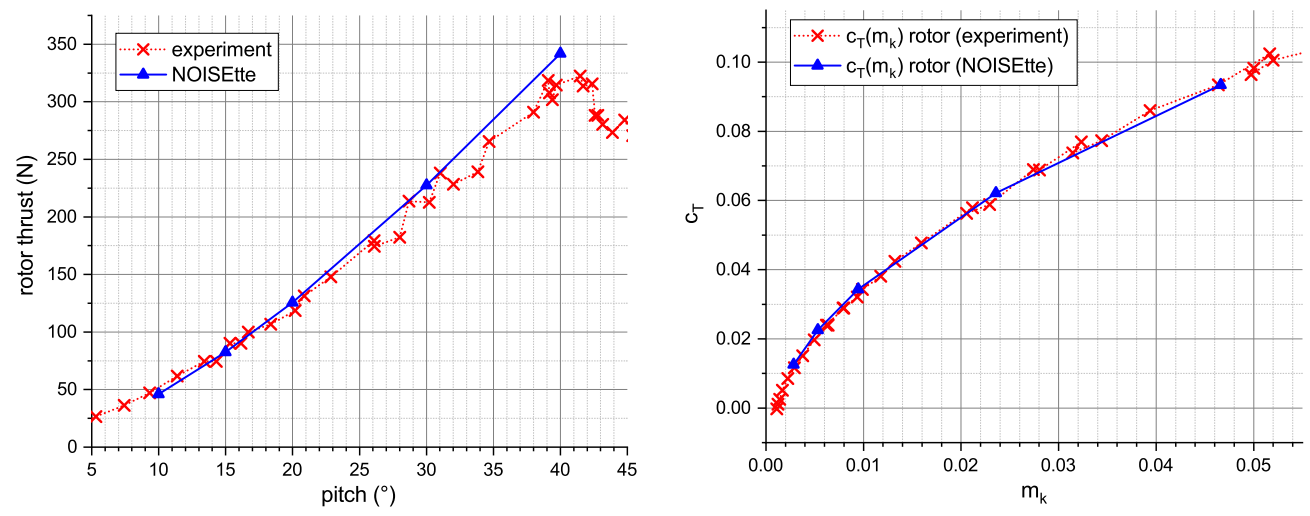

Figure 7: Rotor thrust against pitch angle(left) and rotor thrust coefficient dependence on the torque coefficient

$0.2 \mathrm{~m}$, respectively. In both physical and numerical experiments the rotor rotation speed was 360RPM, which corresponds to the blade tip velocity $45.24 \mathrm{~m} / \mathrm{s}$. Three regimes with different flight velocities 6.79 , 11.31 , and $20.36 \mathrm{~m} / \mathrm{s}$ at zero rotor angle of attack were studied. The value of the Reynolds number evaluated by the chord of the blade and its tip velocity was $\operatorname{Re}=0.455 \times 10^{6}$.

The general flow pattern is mainly determined by the tip vortices shed from the blades, convected downstream, and interacting with both the blades and the vortex structures occurring in the flow. The locations of the cores of the tip vortices repeat the trajectories of the blade tip motions with account for rotor ro-

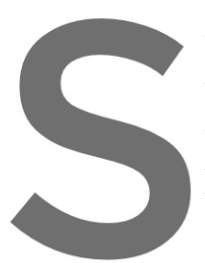
tation and the oncoming the Q-criterion isosurface the rotor rotation plane $x O$

Fig. 8 also presents the
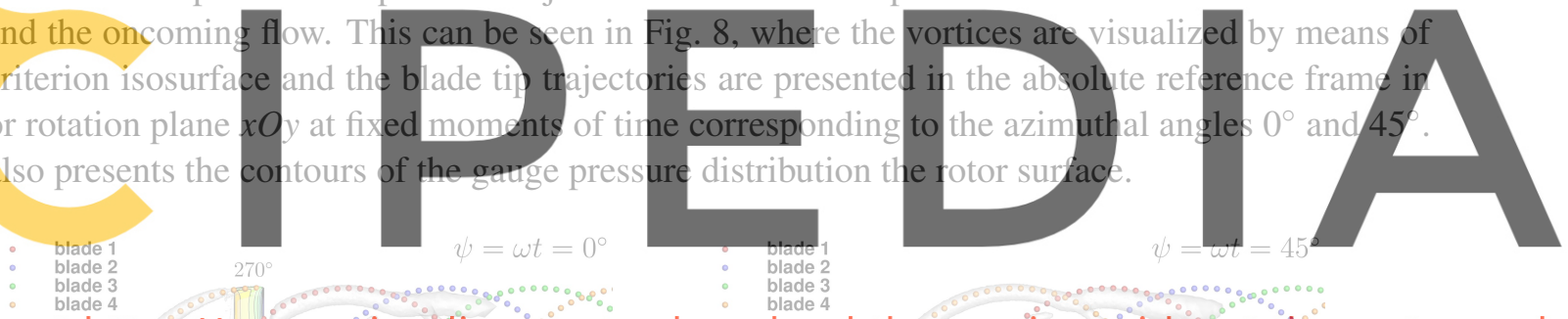

Register for free at https//www.scipedia.com to download the version without the watermark

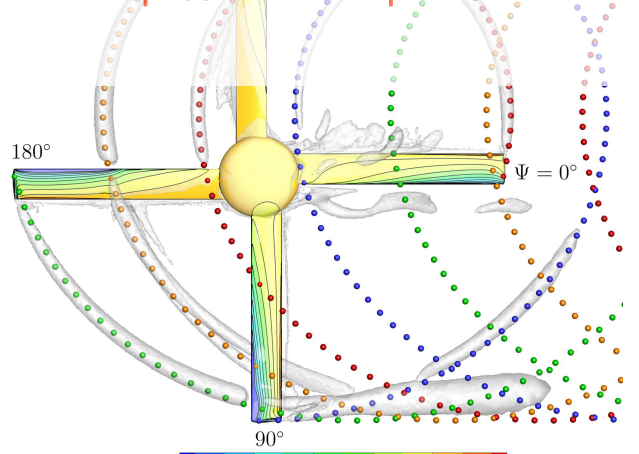

$p_{\mathrm{g}}:-50000-38000-26000 \quad-14000 \quad-2000 \quad 10000$

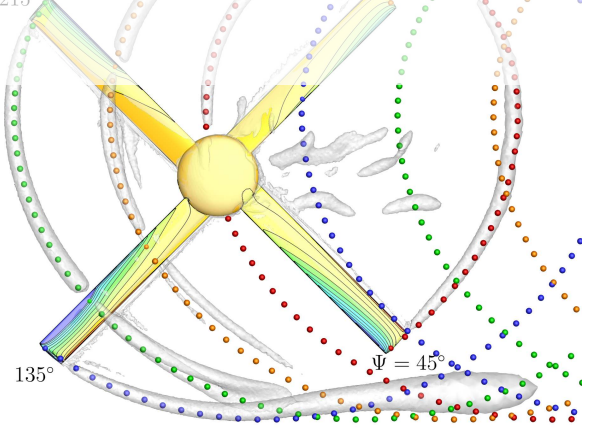

$p_{q}:-50000-38000-26000-14000 \quad-2000 \quad 10000$

Figure 8: Tip vortices trajectories: blade tips trajectories with $Q$-criterion isosurfaces for azimuth $0^{\circ}$ (left) and $45^{\circ}$ (right)

The pressure coefficient distributions in different sections of the rotor blades obtained in the numerical 
experiments using the NOISEtte and ANSYS CFX are presented on Fig. 9. The numerical results are in good agreement with each other on the entire blade chord, except from slight disagreements in the zone of the blade's trailing edge due to different resolutions of the computation meshes in this mesh region.
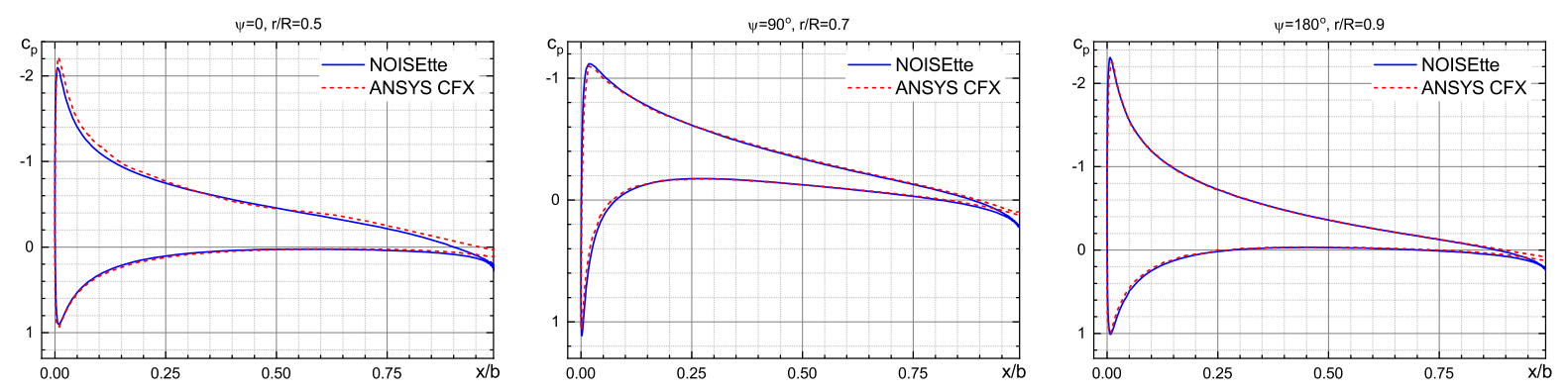

Figure 9: Pressure coefficient distribution along the blade chord calculated using NOISEtte and ANSYS CFX at different span-wise locations and azimuthal angle

The detailed problem setup and results analysis presented in the [15].

\section{CONCLUSION}

The paper presents the method we develop for the numerical simulation of a turbulent flow of viscous compressible gas near a rigid helicopter rotor under various operating conditions. The method allows to efficiently perform predictions on hybrid unstructured meshes. It is implemented in the in-house code NOISEtte software package. A feature of the numerical method is the use of the original EBR schemes. These schemes provide accuracy higher than the traditional Godunov-type finite-volume methods at computational costs significantly lower with respect to the very high order methods. The usage of hybrid unstructured meshes makes it easier to treat the geometry of real helicopter rotors. The parallel implementation of the whole numerical algorithm is based on a hybrid MPI-OpenMP model and makes it possible to use computer systems with hundreds of thousands of CPU cores with high efficiency of parallelization.

The validation of the developed numerical method and code NOISEtte is carried out on by simulating several cases considering helicopter rotors in hovering mode with axial flow and in the forward flight mode. The obtained numerical results show a good agreement with the available experimental data. The developed method is applicable not only for simulating the flow near helicopter rotors. It can be used, for instance, to study numerically the aerodynamics of rotary-wing aircraft (converters, quadcopters, wind power plants) and turboprop aircraft.

This work has been carried out using computing resources of the federal collective usage center Complex for Simulation and Data Processing for Mega-science Facilities at NRC "Kurchatov Institute", http://ckp.nrcki.ru/.

\section{REFERENCES}

[1] Gorobets A. Parallel Algorithm of the NOISEtte Code for CFD and CAA Simulations Lobachevskii Journal of Mathematics (2018) 39:4:524-532 — DOI: 10.1134/S1995080218040078.

[2] Garipova L., Batrakov A., Kusyumov A., Mikhaylov S., Barakos G. Aerodynamic and acoustic 
analysis of helicopter main rotor blade tips in hover International Journal of Numerical Methods for Heat and Fluid Flow (2016) 26:7:2101-2118 — DOI: 10.1108/HFF-08-2015-0348.

[3] Kopiev V.F., Zaytsev M. Y., Vorontsov V. I. Karabasov S. A., Anikin V. A. Helicopter noise in hover: Computational modelling and experimental validation Acoust. Phys. (2017) 63:6:686698 - DOI: $10.1134 /$ S1063771017060070.

[4] Ricci F., Silva P. A. S. F., Tsoutsanis P. and Antoniadis A. F. Hovering rotor solutions by highorder methods on unstructured grids Aerospace Science and Technology (2020) 97:105648 — DOI: $10.1016 / \mathrm{j}$.ast.2019.105648

[5] Abalakin I., Bakhvalov P. and Kozubskaya T. Edge-based reconstruction schemes for unstructured tetrahedral meshes International Journal for Numerical Methods in Fluids (2015) 81:6:331-356 — DOI: 10.1002/fld.4187.

[6] Bakhvalov P., Kozubskaya T. EBR-WENO scheme for solving gas dynamics problems with discontinuities on unstructured meshes Computers and Fluids (2017) 157:312-324 _ DOI: 10.1016/j.compfluid.2017.09.004.

[7] Abalakin I. V., Anikin V. A., Bakhvalov P. A., Bobkov V. G. and Kozubskaya T. K. Numerical investigation of the aerodynamic and acoustical properties of a shrouded rotor Fluid Dynamics (2016) 51(3):419-433 — DOI:10.1134/s0015462816030145.

[8] Bakhvalov P. A., Kozubskaya T. K. Construction of edge-based 1-exact schemes for solving the Euler equations on hybrid unstructured meshes Computational Mathematics and Mathematical Physics (2017) 57:4:680-697 — DOI: 10.1134/s0965542517040030.

[9] Duben A. P., Kozubskaya T. K. Evaluation of Quasi-One-Dimensional Unstructured Method for Jet Noise Prediction AIAA Journal (2019) 57:12:5142-5155 — DOI: 10.2514/1.j058162.

[10] Abalakin I. V., Bobkov V.G. and Kozubskaya T. K. Implementation of the low Mach number method for calculating flows in the NOISEtte software package Math. Models Comput. Simul. (2017) 9:688-696 — DOI: 10.1134/S2070048217060023.

[11] Caradonna F. X., Tung C. Experimental and analytical studies of a model helicopter rotor in hover NASA Technical Memorandum 1981-81232 (1981) NASA, Ames Research Center

[12] Kocurek J. D., Tangler J. L. A Prescribed Wake Lifting Surface Hover Performance Analysis Journal of the American Helicopter Society (1977) 22:1:24-35 — DOI: 10.4050/JAHS.22.24.

[13] Abalakin I. V., Bahvalov P. A., Bobkov V. G., Kozubskaya T. K., Anikin V. A. Numerical simulation of aerodynamic and acoustic characteristics of a ducted rotor Mathematical Models and Computer Simulations (2016) 8:3:309-324 — DOI: 10.1134/s2070048216030030.

[14] Abalakin I. V., Anikin V. A., Bakhvalov P. A., Bobkov V. G., Kozubskaya T. K. Numerical investigation of the aerodynamic and acoustical properties of a shrouded rotor Fluid Dynamics (2016) 51:3:419-433 — DOI: 10.1134/S0015462816030145.

[15] Abalakin I. V., Bobkov V. G., Kozubskaya T. K., Vershkov V. A., Kritsky B. S., Mirgazov R. M. Numerical Simulation of Flow around Rigid Rotor in Forward Flight Fluid Dynamics (2020) 55:4:534-544 — DOI: 10.1134/S0015462820040011. 